\title{
Disentangling telluric lines in stellar spectra
}

\author{
P. Hadrava
}

Astronomický ústav, Akademie věd České republiky, 25165 Ondřejov, Czech Republic

Received 15 September 2005 / Accepted 28 October 2005

\section{ABSTRACT}

The use of a method of spectra disentangling for telluric lines is explained in detail, with a particular emphasis on high-precision radial-velocity measurements for the search for extrasolar planets. New improvements to the method are introduced.

Key words. atmospheric effects - techniques: spectroscopic - techniques: radial velocities - line: profiles - stars: atmospheres stars: planetary systems

\section{Introduction}

Ground-based spectroscopy of astrophysical objects is influenced by telluric lines, which are often pronounced especially in the red and infrared region. These lines have to be taken into account for numerous reasons, the most important of which are: (i) the need to clean the stellar spectra of this additional absorption, (ii) the possibility to check or to improve the definition of the wavelength scale in the spectra (see, e.g., Griffin \& Griffin 1973; Greimel \& Yang 1999) and (iii) a study of the Earth's atmosphere (cf. Griffin 2005). While in the first case the telluric absorption is harmful, in the other two cases it is useful. For the purpose (ii) not only are the natural atmospheric lines used but artificially generated lines from hydrogen-fluoride, iodine or other absorption cells (cf. e.g. Campbell 1983; Butler et al. 1996) can also be imprinted on the observed spectra. Because the problems of the data processing methods discussed here for absorption cells and atmospheric lines are in principle equivalent, we shall refer to both cases as the "telluric lines".

In the above items it is necessary to accurately separate the telluric lines from the ("stellar") spectrum of the observed background object. Several methods have been developed for this purpose with different advantages and limitations. In principle, the telluric lines can be distinguished from the stellar lines using a proper model of their wavelengths, ratios of strengths and line profiles (cf. e.g. Adelman et al. 1996) or by their variability in wavelength (due to the annual motion of the Earth) or in strength (due to variations of the air mass and humidity).

The method of disentangling spectra of multiple stars (Simon \& Sturm 1994; Hadrava 1995, P1 hereafter) generalized for variable line-strengths (Hadrava 1997, P2 hereafter) is suitable to separate telluric lines. This possibility has been incorporated in the code KOREL for Fourier disentangling
(Hadrava 2004b, P3 hereafter) and has been widely used (e.g. Harmanec et al. 1997 etc.).

Hrudková \& Harmanec (2005, HH hereafter) applied the KOREL disentangling of telluric lines to $\alpha$ Boo and advertised its usefulness for studies of late-type single stars. Unfortunately, they had not properly taken into account explanations of the method (Hadrava 2004a or P3) and give some misleading information. To prevent further confusion we give the explanation more explicitly here. In addition, some new possibilities and improvements of the method are presented.

\section{Disentangling of telluric lines using KOREL}

The disentangling of spectra is made possible by the variations of mutual Doppler shifts, and thus works equally for different components of a multiple stellar system or for telluric lines moving annually with respect to single or multiple stars. Telluric, interstellar and binary spectra can be disentangled simultaneously. However, because the telluric lines also vary in strength, their disentangling became possible in practice only after a generalization enabling one to take into account that line-strength variation (cf. P2). On the other hand, this variability in strength is a helpful feature in disentangling telluric lines.

In the first applications, the orbital parameters of telluric lines were converged by the disentangling method. However, because these parameters are given by the annual motion of the Earth and by the ecliptic coordinates of the observed object, which are known, it is advantageous to use the constraints on these parameters as facilitated by the code PREKOR (cf. P3 for details). The observed stellar spectra are usually reduced to the heliocentric wavelength scale. In this case the fictitious centre of mass of the Earth's relative motion (i.e. orbit No. 3 if the telluric lines are identified with component No. 5 - cf. Fig. 1 in P1) with respect to the star or stellar system is put close to 
the star, imposing a very small $K$-velocity of the star, and a proportionally small value of the mass ratio $q_{3} \equiv m_{\text {Earth }} / m_{\text {star }}$ is chosen to give the proper amplitude $K_{\text {Earth }}$ of the telluric lines. The value $K_{\text {star }}=1 \mathrm{~m} / \mathrm{s}$ is preset and the corresponding value of $q_{3}$ is calculated from the coordinates of the star by the code PREKOR, but users may rescale both these numbers from PREKOR to keep $K_{\text {star }}$ negligible if a higher accuracy is desirable. This can be necessary especially in applications that search for extrasolar planets or other low-mass companions, when the lines of an absorption cell can also be superimposed on the stellar spectrum to provide a more precise wavelength scale (as mentioned in Sect. 1). However, for such studies requiring high precision, the Keplerian orbit is an insufficient approximation for the velocity of the Earth and a more accurate approximation, which takes into account corrections for other planets in the Solar system, has to be involved. For this purpose the PREKOR code includes the subroutine kindly provided by C. Ron and J. Vondrák (Ron \& Vondrák 1986) which calculates the vector of the Earth's velocity with a precision of $0.02 \mathrm{~m} / \mathrm{s}$. The disentangling can be then performed with the velocities of the telluric iodine lines unbound to a Keplerian orbit (as described in P3) but fixed by this more precise model. Some differences between radial velocities of telluric lines in individual exposures found by KOREL and the Ron and Vondrák model can be caused by instrumental or data-processing errors in determining the wavelength scale. The whole spectrum can be then shifted by a new run of PREKOR. In principle, a version of the disentangling method with self-adjustment of wavelengths could be developed for cases with well-defined telluric/iodine lines in all spectra.

An alternative possibility is to disentangle the spectra in instrumental, instead of in heliocentric, wavelengths, to set the $K$-velocity of the whole observed stellar system equal to the corresponding projection of the Earth's velocity in the ecliptic on the line of sight, and to formally set the mass ratio $m_{\text {Earth }} / m_{\text {star }}$ very large to decrease the $K$-velocity of telluric lines safely below the resolution capability of the spectra.

\section{The role of line-strength variability}

It is claimed in $\mathrm{HH}$ that "the disadvantage of the method is that one needs a number of spectra of the star in question, secured at different months of the year" to disentangle telluric lines from spectra of a single star. It is not necessarily the case, as can be demonstrated by the example shown in Fig. 1. The three Ondřejov RETICON spectra in the region of $\mathrm{H}_{\alpha}$ line of the star $\tau$ Boo, known for spectroscopic observations of its planet, were taken within three days. To exclude the possibility that the disentangling of telluric lines is due either to the annual change of heliocentric correction of the radial velocity (which is about $0.6 \mathrm{~km} \mathrm{~s}^{-1}$ between the first and the third exposure) or to the motion of the star itself (which has a period of about 3 days and is detectable by disentangling a larger set of spectra), the times of all three exposures were set equal to the time of the middle one. Nonetheless, the disentangling is satisfactory. This is owing to the differences in strengths of the telluric lines, which are obviously weakest in the second and strongest in the third spectrum. The difference between two

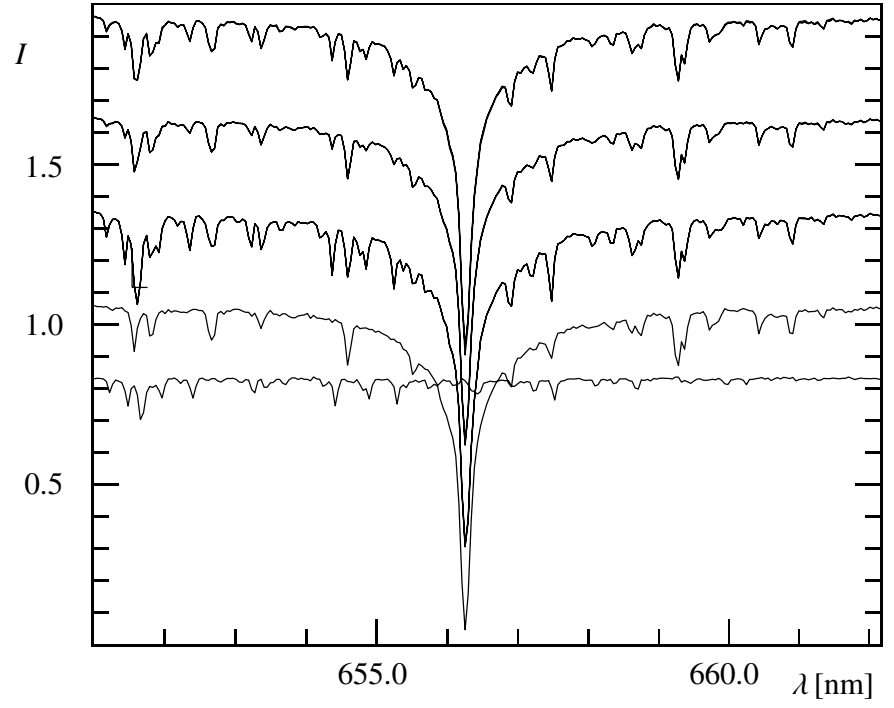

Fig. 1. The standard KOREL output for three spectra of $\tau$ Boo (upper three lines) taken on three consecutive nights disentangled as stellar (the fourth line) and telluric spectra (bottom line).

chosen spectra with different strengths determines the shape of the telluric spectrum $I_{\text {tell }}$ (in some reference scale), which must be then subtracted from each exposure $I_{\mathrm{obs}}(t)$ with a proper multiplicative line-strength factor $s(t)$ to get the stellar spectrum $I_{0}$,

$I_{0}=I_{\mathrm{obs}}(t)-s(t) I_{\mathrm{tell}}$.

The solution of factors $s(t)$ is not unique, because $k s(t)(k>0)$ gives the same solution with the rescaled telluric spectrum $I_{\text {tell }} / k$ and $s(t)-\Delta s$ fits the observed spectra equally by the stellar spectrum $I_{0}+\Delta s I_{\text {tell }}$. The strengths $s(t)$ thus cannot be solved directly from Eq. (7) in P2 or Eq. (30) in P3, which is singular in this case, but can be converged by a simplex method which finds some of the existing solutions. By fixing some of the strengths we can choose the proper solution, or it can be more easily found by KOREL using even relatively small seasonal differences in heliocentric radial-velocity corrections (either for stars close to the poles of the ecliptic or for data covering a short part of the year). An important condition which is worth satisfying during the observation is thus obtaining spectra at different air masses. (Our early experience confirms the seemingly paradoxial fact that the disentangling of telluric spectra significantly improves if spectra that are highly blended with telluric lines are included in the solution.)

Telluric lines consist of several subsystems whose strength may vary independently. Typically, lines of $\mathrm{O}_{2}, \mathrm{~N}_{2}$ and $\mathrm{Ar}$ depend mainly on the air mass, while lines of $\mathrm{H}_{2} \mathrm{O}$ depend also on the instantaneous weather (cf. Adelman et al. 1996) and the $\mathrm{O}_{3}$ lines should be checked for long-term variations (Griffin 2005). Because these lines have identical radial velocities, they can be disentangled only by their variable strengths. In KOREL, it is possible by the above explained method to assign the annual motion of the Earth to both orbits Nos. 2 and 3 (and a negligible $K$-velocity to orbit No. 1), so that all the components in Nos. 3, 4 and 5 can correspond to telluric lines (cf. 
Fig. 1 in P1). However, to separate the subsystems, good initial estimates of their strengths are needed, or spectra of at least some of them should be constrained by an accurate model, as explained below. An alternative method, introduced by Lynch (2005, cf. Lynch \& Polidan 1997) to separate spectral components that change their line-strengths independently, could be used for exposures with negligible differences in radial velocities between the stellar and telluric lines. This also confirms that the limitation claimed by HH is incorrect. Lynch's method is based on the SVD/PCA technique and indicates how many independent components (characterized by their strengths) are present in the data, while this number must be fixed in the present versions of disentangling. Combination of both methods can thus be useful for the problems in question.

\section{Non-linearity of telluric lines}

$\mathrm{HH}$ mentioned as a "principal problem when KOREL is used to disentangle telluric lines" the question of nonlinearity (Hensberge 2001). HH then incompletely rephrased the original answer given by the present author and ignored the recent, more thorough explanations (Hadrava 2004a or P3). The "problem" is that the telluric spectrum is not an additive, but a multiplicative component in the observed spectra, i.e. the observed spectrum is (cf. Eq. (31) in P3)

$I_{\text {obs }}(x, t)=\exp (-\tau(x, t)) I_{0}(x, t)$,

where $I_{0}$ is the spectrum of the studied object as seen outside the Earth's atmosphere and $\tau$ is the optical depth along the line of sight in the atmosphere. This can be expressed as a sum of $I_{0}$ and a negative telluric component:

$I_{\text {tell }}=\left(\mathrm{e}^{-\tau}-1\right) I_{0} \simeq-\tau I_{0}$,

which also depends on $I_{0}$. Consequently, the depth of the telluric line in Fig. 1 which falls close to the centre of the stellar $\mathrm{H}_{\alpha}$ line is underestimated in the decomposed spectrum relative to the depths of telluric lines falling on the continuum. If the disentangled spectra are to be used for spectroscopic studies of the atmosphere of the Earth, the telluric spectrum must be divided by the mean stellar spectrum. Such a renormalisation has also to be applied if we want to use the telluric spectrum disentangled from one star as a template for constrained disentangling of another star, as will be explained later. The only discrepancy arises if the telluric line moves (either due to its own seasonal shifts or due to the orbital motion of the star) in the part of stellar line-profile with a steep slope, or if the strength of the line varies, e.g. in an eclipse of the star. The error in the residuals $(\mathrm{O}-\mathrm{C})$ is proportional to the depth of the telluric line multiplied only by the difference in the local depth of the stellar line from its mean value, not its whole depth. Possible deflection of the decomposed spectrum is generally even smaller.

In the simple case of a one-component stellar system (either a single star or a multiple system dominated by the spectrum of one component) plus a telluric spectrum the problem of nonlinearity of the telluric component can be avoided by a logarithmic transformation of the (rectified) input spectra:

$$
\begin{aligned}
I(t, x) \rightarrow I^{\prime}(t, x) & =1+\ln I \\
& =1+\ln I_{0}(t, x)-\tau(t, x),
\end{aligned}
$$

where $I_{0}$ is the stellar spectrum and $\tau$ is the monochromatic optical depth of the Earth's atmosphere (cf. Eq. (31) in P3). The corresponding backward transformation should be applied to the disentangled spectra. This would not be appropriate to disentangling standard binaries (for which the spectra are additive, $\left.I_{0}=I_{1}+I_{2}+\ldots\right)$. However, in searching for a faint component $I_{2}$ in the presence of strong telluric lines in the spectrum $I_{1}$ of the dominant component, it can be advantageous to use the linearization $\ln I_{0}=\ln I_{1}+\left(I_{2}+\ldots\right) / I_{1}$ and to disentangle the spectra on a logarithmic scale of intensities. Naturaly, the normalization of disentangled telluric spectrum described in the previous paragraph must not be performed if the spectra are transformed into a logaritmic scale of intensity. Instead, the inverse transforms of both the telluric and the stellar spectra have to be applied.

\section{Solution instabilities and use of constraints}

Despite the solutions described above, some problems may appear or increase when telluric lines are included in the disentangling. One example is the problem of waves in the disentangled spectra $I_{j}(x)$, due to instabilities of the lowest Fourier modes $\tilde{I}_{j}(y)$, for which the corresponding equations (Eqs. (5) in P1, (6) in P2, (20) or (28) in P3) of general form

$$
\begin{array}{r}
\sum_{j=1}^{n}\left[\sum_{l=1}^{N} w_{l} \tilde{\Delta}_{j}\left(y, t_{l}, p\right) \tilde{\Delta}_{m}^{*}\left(y, t_{l}, p\right)\right] \tilde{I}_{j}(y) \\
=\sum_{l=1}^{N} w_{l} \tilde{I}\left(y, t_{l}\right) \tilde{\Delta}_{m}^{*}\left(y, t_{l}, p\right)
\end{array}
$$

are nearly singular (here $I$ are the spectra observed at time $t_{l}$, $\Delta_{j}$ are broadening functions for component $j-$ cf. P1, P2, P3 for details). These instabilities often increase with a new component such as a telluric spectrum, at least until the appropriate strengths or orbital parameters are well approximated. Possible errors in rectification or real non-Doppler variations of stellar spectra may force the solution to use its new freedom to fit these features instead of the true telluric spectrum. Two hints are recommended in this case: (i) determine the strengths of telluric lines from a spectral region where they are dominant (e.g. the short-wavelength region shown in Fig. 1) and fix them in other regions at least until the other parameters are not iterated further, or (ii) use the fact that the telluric lines are mostly narrow, i.e. contained in higher Fourier modes, and filter out the lowest modes at least until the correct solution is approached in higher modes.

Quite often some problems caused by the rectification, lineprofile variability or an unresolved component of the spectrum persist in centres of deep stellar lines and a small contribution to those lines then appears in the telluric spectrum, which is otherwise reasonable (with the correct lines on a flat continuum) in the far wings of the stellar lines and in the stellar continuum. In such a case, use of a recently developed "constrained disentangling" procedure can be helpful. This will be described elsewhere (Hadrava 2005). For our present purpose 
it is necessary to know that the observed spectra can be approximated by the form

$$
\begin{aligned}
I(x, t)= & \sum_{j=1}^{m} I_{j}(x) * \Delta_{j}(x, t, p) \\
& +\sum_{j=m+1}^{n} J_{j}(x) * \Delta_{j}(x, t, p),
\end{aligned}
$$

where the first sum includes the component spectra $I_{j}$ which are unknown and have to be isolated by the disentangling and the second one includes those spectra which are known and constrained by some templates $J_{j}$ (all component spectra being Doppler shifted or broadened by broadening functions $\Delta_{j}$, the parameters $p$ of which may be also solved by disentangling). The shape of the telluric spectrum can thus be constrained in this sense, either removing the residuals of stellar lines from the preliminarily disentangled spectra by their additional rectification, or directly by using a template for the telluric spectrum obtained from solutions for other stars (with the abovementioned renormalization). With these constrained telluric spectra, the solution may be forced to divide the previous artifacts between the different disentangled stellar spectra (and to give better mean line-profiles), or to put the discrepancies into residual spectra, from where their nature can be judged.

Acknowledgements. The author would like to thank the referee, Elizabeth Griffin, for helpful comments. The inspiring discussions with D. Holmgren, J. Kubát and D. Lynch are also acknowledged.
Thanks are due to colleagues C. Ron and J. Vondrák for providing their procedure for calculation of the Earth's velocity. The observations of $\tau$ Boo by H. Božić are also highly appreciated. This work has been supported by project AV0Z10030501.

\section{References}

Adelman, S. J., Gulliver, A. F., \& Holmgren, D. E. 1996, in Model atmospheres and spectrum synthesis, ed. S. J. Adelman, F. Kupka, \& W. W. Weiss, ASP. Conf. Ser., 108, 293

Butler, R. P., Marcy, G. W., Williams, E., et al. 1996, PASP, 108, 500 Griffin, R. E. 2005, PASP, 117, 885

Griffin, R. E., \& Griffin, R. F. 1973, MNRAS, 162, 243

Greimel, R., \& Yang, S. L. S. 1999, in Precise radial velocities, ed. J. B. Hearnshaw, \& C. D. Scarfe, ASP. Conf. Ser., 185, 48

Hadrava, P. 1995, A\&AS, 114, 393 (P1)

Hadrava, P. 1997, A\&AS, 122, 581 (P2)

Hadrava, P. 2004a, in Spectroscopically and spatially resolving the components of the close binary stars, ed. R. W. Hilditch, H. Hensberge, \& K. Pavlovski, ASP. Conf. Ser., 318, 86

Hadrava, P. 2004b, Publ. Astron. Inst. ASCR, 92, 15 (P3)

Hadrava, P. 2005, Ap\&SS, submitted

Harmanec, P., Hadrava, P., Yang, S., et al. 1997, A\&A, 319, 867

Hensberge, H. 2001, private communication

Hrudková, M., \& Harmanec, P. 2005, A\&A, 437, 765 (HH)

Lynch, D. E. 2005, Spectral Decomposition of Time Variable Sources (unpublished)

Lynch, D. E., \& Polidan, R. S. 1997, AAS, 190, 2804

Ron, C., \& Vondrák, J. 1986, Bull. Astron. Inst. Czechosl., 37, 96

Simon, K. P., \& Sturm, E. 1994, A\&A, 281, 286 